\title{
DINÁMICA Y CONTROL DE MALEZAS EN PEJIBAYE PARA PALMITO (Bactris gasipaes K.) ${ }^{1}$
}

\author{
Antonio Bogantes ${ }^{2}$, Renán Agüero ${ }^{3}$
}

\begin{abstract}
RESUMEN
Dinámica y control de malezas en pejibaye para palmito (Bactris gasipaes K.). Este experimento se realizó en la Estación Experimental Los Diamantes, ubicada en Guápiles, Costa Rica. Se determinó el efecto de cuatro distancias de siembra entre plantas $(0,25 ; 0,50 ; 0,75 ;$ y $1,00 \mathrm{~m})$ y dos estrategias de control (física y química) sobre la dinámica de malezas en una plantación de pejibaye sin espinas para palmito. Las variables evaluadas fueron cobertura y altura de las malezas, especies de malezas presentes y daño causado por el herbicida a las malezas y plantas. La mecanización promovió la germinación de especies presentes en el banco de semillas. Se encontraron 31 especies de malezas en un total de 3456 $\mathrm{m}^{2}$ durante los primeros 14 meses después de la siembra (mds). Hubo interacción entre distancias de siembra y estrategias de control con respecto a cobertura de poaceas y de malezas de hoja ancha 14 mds. La especie Paspalum fasciculatum dominó en las parcelas con control físico, mientras que Ageratum conyzoides fue dominante en las parcelas con control químico. La altura y la cobertura total de las malezas en las bandas y en los centros de las filas varió según la distancia de siembra entre plantas de palmito nueve mds. El control químico de malezas con glifosato fue bueno, salvo algunas excepciones especiales en la primera y cuarta aplicación, por el tamaño de las malezas o falta de intercepción del herbicida.
\end{abstract}

\begin{abstract}
Weed dynamic and control in peach palm (Bactris gasipaes k.) for palm hearts. The study was conducted at Los Diamantes Experiment Station, located in Guapiles, Costa Rica, to compare four sowing densities of thornless palms and two weed management strategies (chemical and physical) on weed population dynamics of this crop, during the first year of growth. Weed cover and height, species present and herbicide damage were among the variables recorded. Soil tillage prior to the crop establishment promoted weed germination from the seed bank. A total of 31 weed species were recorded from a $3452 \mathrm{~m}^{2}$ experimental area during the first 14 months after sowing. An interaction between sowing distance and weed management strategies was determined for gramineus and broadleaf species. Paspalum fasciculatum was the dominant species in plots under hand weeding while Ageratum conyzoides prevailed in glyphosate treated plots. Nine months after sowing, weed height and cover along crop rows or between them were different depending on the sowing density. In general, chemical weed control was efficient, although care should be taken during the first spraying when crop and weed height are similar, to avoid crop damage.
\end{abstract}

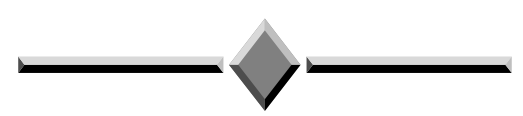

\section{INTRODUCCIÓN}

El pejibaye para palmito se siembra en Costa Rica desde 1974 y el área de siembra aumentó hasta aproxi- madamente 12.000 ha en 1998 , constituyéndose en una alternativa importante para exportación.

La planta de pejibaye se reproduce principalmente por semilla sexual, la cual se origina por polinización

1 Recibido para publicación el 16 de mayo del 2002.

2 Estación Experimental Los Diamantes. MAG (Costa Rica). Teléfono (506) 710-7853, fax 710-7854. Dirección electrónica: magdiama@racsa.co.cr

3 Centro de Investigaciones en Protección de Cultivos. U.C.R. teléfono (506) 207-3446. Dirección electrónica: raguero@cariari.ucr.ac.cr. 
cruzada. Por lo anterior, desde las primeras siembras establecidas, las poblaciones utilizadas para palmito han presentado gran variabilidad genética y se han caracterizado en su mayoría por la presencia de espinas. Recientemente se han seleccionado plantas sin espinas, las cuales han sido puestas a disposición de los productores (Mora et al. 1999).

Al inicio las distancias de siembra en pejibaye con espinas para palmito eran altas (tres $m$ entre hileras y un $m$ entre plantas); sin embargo, se notó inadecuada porque el espacio ocupado por cada planta era excesivo. Posteriormente se aumentó la densidad, de manera que la distancia $2 \mathrm{~m}$ x $1 \mathrm{~m}$ ha sido la más común en los últimos 10 años. Factores como el mercado, adaptaciones en el uso de maquinaria y las nuevas variedades podrían provocar nuevos cambios en esta práctica (Mora 1989).

El uso de variedades sin espinas permite aumentar la densidad y en ese sentido, el uso de altas densidades de siembra también podría ayudar a disminuir la incidencia de malezas, lo cual facilitaría el manejo del cultivo.

Con respecto al combate de malezas en palmito, el principal esfuerzo se hace durante el primer año. En este período éstas representan menor competencia al cultivo por luz y espacio debido al poco desarrollo de las plantas de pejibaye, lo cual demanda el uso de una o más estrategias de combate.

En una prueba preliminar, cuyos datos aún no se publican, los autores del presente estudio determinaron una reducción del 93,3\% en el peso fresco de plantas de palmito que crecieron durante 4,5 meses en libre competencia con las malezas.

El manejo de las malezas se ha limitado al combate físico, principalmente chapea manual o mecánica y al control químico con herbicidas tradicionales. Sin embargo, por razones económicas y ecológicas el concepto moderno de manejo consiste en reducir el efecto nocivo de las malezas en vez de suprimirlas, tratando de evitar un desbalance biológico que perjudique el cultivo. Estos sistemas han evolucionado desde el control manual hasta el biológico, pero para una correcta utilización es importante entender la relación de las malezas con el cultivo, las prácticas de producción y el ambiente (Pitty y Muñoz 1991).

El objetivo del presente trabajo fue evaluar el efecto de cuatro distancias de siembra y dos estrategias de control (física y química) sobre la dinámica de población de las malezas en una plantación de pejibaye sin espinas para palmito.

\section{MATERIALES Y MÉTODOS}

\section{Descripción del área de investigación}

El estudio se realizó entre marzo del 2000 y mayo del 2001, en la Estación Experimental Los Diamantes propiedad del Ministerio de Agricultura y Ganadería, ubicada en Guápiles, cantón Pococí, provincia de Limón; a una altitud de $249 \mathrm{~m}$, con temperatura mínima promedio de $24{ }^{\circ} \mathrm{C}$ y máxima promedio de $29{ }^{\circ} \mathrm{C}$. La precipitación promedio anual es de $4.500 \mathrm{~mm}$.

Según Valverde y Ugalde (1990), la mayor parte de la finca $(75 \%)$ está sobre un abanico aluvial muy grueso, con pedregones en la parte superior y más fino en la sección terminal. Los sedimentos son de origen volcánico, de composición andesítica.

\section{Preparación del terreno y siembra}

Se dio una pasada de arado y dos de rastra y se sembró en marzo del 2000. Se usaron plantas con raíz desnuda de pejibaye sin espinas de la raza Diamantes 10 (Yurimaguas), con tres meses de germinadas y dos pares de hojas formadas. Los hoyos se hicieron con espeque y fue necesaria la resiembra de plantas 1,4 y 8 semanas después de la siembra.

La fertilización se hizo siguiendo las instrucciones hechas en el manual de recomendaciones del cultivo (Bogantes 1996).

Previo al inicio de los tratamientos de control de malezas (mayo 2000), se hizo una chapea con cuchillo para homogenizar todas la parcelas.

\section{Tratamientos}

Los tratamientos evaluados fueron la combinación de cuatro distancias de siembra con dos estrategias de manejo de malezas (Cuadro 1).

La estrategia física en las bandas consistió en 10 deshierbas con cuchillo, mientras que en el centro se hicieron dos chapeas con cuchillo y seis con motoguadaña. El control químico se hizo con el herbicida glifosato (Round up 0,40-0,50\% volumen/volumen y Ranger Plus $68 \%$ SG, 0,60\% peso/volumen) según la cobertura y la altura de las malezas en el momento de la aplicación. Se usó una bomba de mochila marca Field King de 14 litros con una boquilla 8002 . 
Cuadro 1. Arreglo de distancias de siembra y estrategias de control de malezas. Guápiles, Limón. Costa Rica. 2000-2001.

\begin{tabular}{cclr}
\hline $\begin{array}{c}\text { Distancia/ } \\
\text { hilera (m) }\end{array}$ & $\begin{array}{c}\text { Distancia/ } \\
\text { planta (m) }\end{array}$ & $\begin{array}{c}\text { Manejo } \\
\text { malezas }\end{array}$ & $\begin{array}{c}\text { Densidad } \\
\text { (plantas/ha) }\end{array}$ \\
\hline 2,0 & 0,25 & Químico & 20000 \\
2,0 & 0,50 & Químico & 10000 \\
2,0 & 0,75 & Químico & 6666 \\
2,0 & 1,00 & Químico & 5000 \\
2,0 & 0,25 & Fí́sico & 20000 \\
2,0 & 0,50 & Físico & 10000 \\
2,0 & 0,75 & Físico & 6666 \\
2,0 & 1,0 & Físico & 5000 \\
\hline
\end{tabular}

Las aplicaciones a la banda se hicieron en forma dirigida usando una campanola o pantalla según las circunstancias.

Para establecer un control diferenciado, previo a las deshierbas tanto físicas como químicas, se hizo una evaluación de la cobertura y la altura de las malezas en la banda y en el centro; así, durante todo el período de evaluación se permitió una mayor cobertura y altura de malezas en el centro con respecto a la banda. La altura de las malezas en la banda no sobrepasó los 20 centímetros durante las primeras seis semanas, ni los 35 centímetros durante el resto de la evaluación, mientras que en el centro la altura máxima fue 45 centímetros. Durante esos períodos la altura promedio de las plantas fue de $25 \mathrm{~cm}$ para el primero y $50 \mathrm{~cm}$ para el segundo período.

El ancho de la banda fue de $0,25 \mathrm{~m}$ en cada lado de la hilera y en el centro de $1,50 \mathrm{~m}$.

Se usó un diseño de parcela dividida distribuida en cinco bloques al azar. La estrategia de manejo de malezas se asignó a la parcela grande y las distancias de siembra entre plantas a la pequeña para un total de 40 parcelas.

Cada parcela pequeña midió $64 \mathrm{~m}^{2}$ y se usó una área útil de $36 \mathrm{~m}^{2}$; el área experimental total fue de $3.456 \mathrm{~m}^{2}$. Para el análisis estadístico de las variables (análisis de varianza y pruebas de medias) se utilizó el programa SAS.

\section{Variables evaluadas}

\section{Dinámica poblacional}

Antes de la preparación del terreno se hizo una estimación visual de las principales malezas presentes ha- ciendo un recorrido en forma de X por el lote. Posteriormente se hicieron levantamientos de las especies de malezas presentes y su cobertura, uno, seis y catorce meses después de la siembra (mds). Cada levantamiento se hizo con un marco de $0,25 \mathrm{~m}^{2}$ el cual se lanzó cuatro veces por parcela útil en la primera y segunda evaluación y dos veces en la tercera. La estimación de cobertura fue visual, usando la escala propuesta por Soto y Agüero (1992). La identificación de las malezas conocidas, se hizo en el sitio y las no conocidas se recolectaron y se identificaron en el laboratorio de la Estación Experimental Los Diamantes con el apoyo de biólogos del Herbario Nacional.

La variable cobertura por grupos de malezas fue analizada en porcentaje.

\section{Cobertura y altura de las malezas}

Antes de aplicar los tratamientos de control, se hicieron muestreos de cobertura y altura general de las malezas en las bandas y en los centros.

En cada parcela útil, se lanzó dos veces un marco de $0,25 \mathrm{~m}^{2}$ y se hizo una estimación visual de la cobertura en porcentaje. La estimación de la altura de las malezas se hizo en $\mathrm{cm}$. Para decidir sobre la aplicación de los tratamientos de control en la banda o en el centro se consideró la información promedio de las parcelas respectivas. Para el análisis estadístico de la variable cobertura general de malezas en la banda y en el centro se usaron los datos en porcentaje

\section{Daño a las malezas y al cultivo}

Se hicieron evaluaciones visuales del daño causado por el herbicida a las malezas y a las plantas uno y dos semanas después de aplicado el tratamiento herbicida del primero y cuarto ciclo. Se efectuaron dos estimaciones por parcela útil adaptando la metodología de Soto-Agüero (1992) en donde $0=\sin$ daño y $5=$ daño severo (más del $75 \%$ del tejido necrosado). La evaluación a las plantas con daño se hizo en la totalidad de las parcelas.

\section{RESULTADOS}

\section{Total de especies}

Durante la selección y antes de la preparación del terreno se determinó que un $95 \%$ del área estaba cubierta por Paspalum fasciculatum (gamalote) y un 5\% con 
especies de hoja ancha, principalmente Sida sp, Mimosa pudica y Colocasia esculenta. La altura promedio de las malezas fue de $70 \mathrm{~cm}$.

Un mes después de la siembra (1 mds) en toda el área del estudio, se observaron 21 especies de malezas, de las cuales, cuatro eran poáceas, dos ciperáceas y 15 de hoja ancha. A los seis mds la cantidad de especies en el área aumentó a 31 (seis poáceas, cuatro ciperáceas y 21 de hoja ancha) y a los 14 mds se redujo a 24 especies (seis poáceas, dos ciperáceas y 16 de hoja ancha). El total de especies presentes en los diferentes períodos del estudio se describe en el Cuadro 2.

Cuadro 2. Especies de malezas presentes uno, seis y catorce meses después de la siembra. Guápiles, Limón. Costa Rica. 2000-2001.

\begin{tabular}{|c|c|c|c|}
\hline Especies & 1 mes & 6 meses & 14 meses \\
\hline Paspalum conjugatum & $\mathrm{X}$ & $X$ & $\mathrm{X}$ \\
\hline Rottboellia conchinchinensis & $\mathrm{X}$ & & $\mathrm{X}$ \\
\hline Paspalum fasciculatum & $\mathrm{X}$ & $\mathrm{X}$ & $X$ \\
\hline Ischaemum ciliaris & $\mathrm{X}$ & & $\mathrm{X}$ \\
\hline Fimbristylis dichotoma & $\mathrm{X}$ & $\mathrm{X}$ & \\
\hline Cyperus feraz & $\mathrm{X}$ & $\mathrm{X}$ & $\mathrm{X}$ \\
\hline Sida rhombifolia & $\mathrm{X}$ & $X$ & \\
\hline Ageratum conyzoides & $\mathrm{X}$ & $X$ & $\mathrm{X}$ \\
\hline Oxalis barrealieri & $\mathrm{X}$ & $\mathrm{X}$ & $\mathrm{X}$ \\
\hline Colocasia esculenta & $X$ & $X$ & $\mathrm{X}$ \\
\hline Mimosa púdica & $\mathrm{X}$ & $X$ & \\
\hline Commelina difusa & $\mathrm{X}$ & $X$ & $\mathrm{X}$ \\
\hline Physalis angulata & $\mathrm{X}$ & $\mathrm{X}$ & $\mathrm{X}$ \\
\hline Acalypha alopecurioides & $\mathrm{X}$ & & $\mathrm{X}$ \\
\hline Jaegeria hirta & $\mathrm{X}$ & & \\
\hline Emilia fosbergui & $\mathrm{X}$ & $\mathrm{X}$ & \\
\hline Spermacoce lavéis & $\mathrm{X}$ & $\mathrm{X}$ & $\mathrm{X}$ \\
\hline Ludwigia suffruticosa & $\mathrm{X}$ & $\mathrm{X}$ & $\mathrm{X}$ \\
\hline Phyllantus niruri & $\mathrm{X}$ & & $\mathrm{X}$ \\
\hline Alternanthera $\mathrm{sp}$ & $X$ & $X$ & \\
\hline Digitaria sanguinalis & & $\mathrm{X}$ & $\mathrm{X}$ \\
\hline Cenchrus sp & & $\mathrm{X}$ & \\
\hline Eleusine indica & & $X$ & $\mathrm{X}$ \\
\hline Homolepsis sp & & $\mathrm{X}$ & \\
\hline Sida acuta & & $X$ & $\mathrm{X}$ \\
\hline Kyllinga sesquiflora & & $\mathrm{X}$ & $\mathrm{X}$ \\
\hline Lindernia crustácea & & $X$ & \\
\hline Drimaria cordata & & $X$ & $X$ \\
\hline Mollugo verticilata & & $X$ & \\
\hline Phenax sonnerattii & & $\mathrm{X}$ & \\
\hline Spermacoce alata & & $\mathrm{X}$ & $\mathrm{X}$ \\
\hline Mecardonia procumbens & & $X$ & $\mathrm{X}$ \\
\hline Lindernia difusa & & $\mathrm{X}$ & \\
\hline Stachytarpeta cayennensis & & $\mathrm{X}$ & $\mathrm{X}$ \\
\hline Peperomia pellucida & & $\mathrm{X}$ & \\
\hline Cyperus diffusus & & $X$ & \\
\hline Stemodia verticilata & & & $\mathrm{X}$ \\
\hline Pseudoelephantopus spicatus & $\mathrm{X}$ & & $\mathrm{X}$ \\
\hline Total especies & 21 & 31 & 24 \\
\hline
\end{tabular}

$\mathrm{X}=$ presente

\section{Cobertura por grupos}

La cobertura de las malezas poáceas un mds no fue diferente entre estrategias de control $(\mathrm{p} \leq 0,19)$ ni entre distancias de siembra $(\mathrm{p} \leq 0,11)$, no hubo diferencias en la cobertura de especies de hoja ancha entre estrategias de control $(\mathrm{p} \leq 0,08)$ ni entre distancias de siembra $(\mathrm{p} \leq 0,52)$; tampoco hubo diferencias entre estategias $(\mathrm{p} \leq 0,47)$ ni entre distancias $(\mathrm{p} \leq 0,37)$ con respecto a la cobertura de ciperáceas.

Las medias de la cobertura de malezas por grupos según estrategias de control (1 mds) se observan en el Cuadro 3.

Cuadro 3. Porcentaje de cobertura de malezas por grupos en cada estrategia de control, 1 y 6 mds. Guápiles, Limón. Costa Rica. 2000-2001.

\section{Grupo de malezas Estrategia física Estrategia química}

\section{1 mds}

\begin{tabular}{lrl} 
Poáceas & 6,70 & 9,03 \\
Hoja ancha & 7,56 & 9,41 \\
Cyperáceas & 0,30 & 0,41 \\
6 mds & & \\
Poáceas & $90,14 \mathrm{a}$ & $2,57 \mathrm{~b}$ \\
Hoja ancha & $6,31 \mathrm{a}$ & $3,83 \mathrm{~b}$ \\
Ciperáceas & $1,40 \mathrm{a}$ & $0,02 \mathrm{~b}$ \\
\hline
\end{tabular}

No hay diferencias entre medias de la misma fila $1 \mathrm{mds}$, según Duncan $(\mathrm{p}>0,05)$

Medias con letra diferente en la misma fila 6 mds, difieren según prueba Duncan $(\mathrm{P} \leq 0,05)$.

A los seis mds (Cuadro 3), el porcentaje de cobertura de poáceas y ciperáceas fue mayor en las parcelas con control físico con respecto a las del químico ( $\mathrm{p} \leq 0,0001$ y 0,012 respectivamente).

Además, hubo una mayor cobertura de especies de hoja ancha en las parcelas tratadas con herbicida $(p \leq 0,0004)$ y no se dieron diferencias entre distancias para la cobertura de poaceas, hojas anchas y ciperáceas $(\mathrm{p} \leq 0,15 ; 0,17$ y 0,93$)$

No hubo diferencia entre estrategias de control $(\mathrm{p} \leq 0,34)$ ni entre distancias de siembra $(\mathrm{p} \leq 0,11)$ para la cobertura de malezas ciperáceas 14 mds (Cuadro 4).

Con respecto a la cobertura de poaceas y hojas anchas, hubo interacción entre las distancias de siembra con las estrategias de control ( $\mathrm{p} \leq 0,001$ y $\mathrm{p} \leq 0,0001)$; en 
las parcelas con control físico y sembradas a $0,25 \mathrm{~m}$ entre planta, la cobertura de poaceas fue menor que en las parcelas con más distancia entre plantas. Por su parte, en las parcelas con control químico, la cobertura de poaceas fue mayor conforme aumentó la distancia entre plantas, aunque en general la cobertura fue menor si se compara con las parcelas con control físico (Cuadro 4).

En las parcelas con control físico y sembradas a $0,25 \mathrm{~m}$ se dio mayor cobertura de hojas anchas con respecto a las otras distancias; mientras que en la estrategia de control químico hubo menos cobertura en las parcelas a $0,25 \mathrm{~m}$ con respecto a las otras distancias. Con excepción de esas parcelas sembradas a $0,25 \mathrm{~m}$ la cobertura de especies de hoja ancha fue mayor en la estrategia química (Cuadro 4).

\section{Especies dominantes}

La distribución de las especies en la primera evaluación (1 mds), fue muy homogénea en las parcelas de ambas estrategias de control. La mayor cobertura fue de P. fasciculatum (poaceae). A los 6 mds el $83 \%$ de la cobertura total de la especies en las parcelas con control físico correspondió a P. fasciculatum, mientras que, con la estrategia química la cobertura de esa especie fue $0 \%$. En el caso de las parcelas con control químico, la especie de hoja ancha Ageratum conyzoides ocupó el $38 \%$ de la cobertura total de malezas y en segundo lugar Lindernia crustacea con una cobertura del $15 \%$ del total (Cuadro 5).

La dominancia de $P$. fasciculatum en el control físico prevaleció 14 mds acompañada principalmente por Drimaria cordata una especie de hoja ancha. En esa etapa del cultivo también se observó la dominancia de

Cuadro 4. Porcentaje de cobertura de malezas poaceas y de hoja ancha, en cada distancia de siembra, según la estrategia de control, 14 mds. Guápiles, Limón. Costa Rica. 2000-2001.

\begin{tabular}{cccc}
\hline $\begin{array}{c}\text { Estrategia de } \\
\text { control }\end{array}$ & $\begin{array}{c}\text { Distancia entre } \\
\text { planta }(\mathbf{m})\end{array}$ & Poaceas * & Hoja ancha ** \\
\hline \multirow{4}{*}{ Física } & 0,25 & 27,50 & 16,40 \\
& 0,50 & 91,40 & 6,90 \\
& 0,75 & 91,00 & 4,00 \\
& 1,00 & 86,50 & 4,00 \\
\hline \multirow{4}{*}{ Química } & 0,25 & 0,20 & 10,30 \\
& 0,50 & 3,70 & 28,00 \\
& 0,75 & 9,70 & 30,40 \\
& 1,00 & 7,40 & 28,30 \\
\hline Medias diferentes según estrategia de control $(* \mathrm{p} \leq 0,001 \mathrm{y} * *$ \\
$\mathrm{p} \leq 0,0001)$.
\end{tabular}

Cuadro 5. Porcentaje de cobertura promedio de las especies dominantes, en cada estrategia de control 1, 6 y 14 mds. Guápiles, Limón. Costa Rica. 2000-2001.

\begin{tabular}{|c|c|c|}
\hline Mes/especie & Estrategia física & Estrategia química \\
\hline \multicolumn{3}{|l|}{1 mds } \\
\hline Paspalum fasciculatum & 5 & 8 \\
\hline Commelina difusa & 1 & 2 \\
\hline Sida rhombifolia & 2 & 3 \\
\hline Colocasia esculenta & 1 & 1 \\
\hline Total* & 15 & 19 \\
\hline \multicolumn{3}{|l|}{$6 \mathrm{mds}$} \\
\hline Paspalum fasciculatum & 83 & 0 \\
\hline Paspalum conjugatum & 6 & 0 \\
\hline Lindernia crustácea & 1 & 6 \\
\hline Ageratum conyzoides & 1 & 15 \\
\hline Total* & 98 & 39 \\
\hline \multicolumn{3}{|l|}{14 mds } \\
\hline Paspalum fasciculatum & 69 & 0 \\
\hline Paspalum conjugatum & 5 & 1 \\
\hline Drimaria cordata & 7 & 0 \\
\hline Ageratum conyzoides & 0 & 12 \\
\hline Mecardonia procumbens & 0 & 5 \\
\hline Digitaria sanguinalis & 0 & 3 \\
\hline Stemodia verticilata & 0 & 1 \\
\hline Acalypha alopecurioides & 0 & 4 \\
\hline Total $^{*}$ & 82 & 30 \\
\hline
\end{tabular}

* Cobertura del total de las especies según estrategia de control y época de evaluación.

A. conizoides en las parcelas donde se usó el control químico (Cuadro 5).

\section{Cobertura y altura en las bandas y en los centros}

En la primera evaluación ( $2 \mathrm{mds})$, la cobertura general de malezas en la banda fue igual entre estrategias de control $(\mathrm{p} \leq 0,65)$ y entre distancias de siembra $(\mathrm{p} \leq 0,49)$; también la cobertura en los centros fue similar entre estrategias $(\mathrm{p} \leq 0,20)$ y entre distancias $(\mathrm{p} \leq 0,22)$.

Con respecto a la altura de las malezas en la banda, no hubo diferencias entre estrategias ni entre distancias $(\mathrm{p} \leq 0,18$ y 0,81$)$ mientras que la altura de malezas en el centro fue parecida entre estrategias $(p \leq 0,19)$ y entre distancias $(p \leq 0,24)$. En general para este período la cobertura y la altura de las malezas fue menor en la banda que en el centro (Cuadro 6).

La cobertura de malezas 9 mds tanto en la banda $(\mathrm{p} \leq 0,0001)$ como en el centro $(\mathrm{p} \leq 0,0001)$ varió según la distancia entre plantas.

En ambos casos el porcentaje de cobertura fue menor cuando las plantas estaban a $0,25 \mathrm{~m}$ entre sí, y 
Cuadro 6. Porcentaje de cobertura total y altura de malezas $( \pm$ error estandar ) en la banda y en el centro en cada estrategia de control, 2 mds. Guápiles, Limón. Costa Rica. 2000-2001.

\begin{tabular}{lcccc}
\hline Estrategia* & \multicolumn{2}{c}{ Banda } & & \multicolumn{2}{c}{ Centro } \\
\cline { 2 - 3 } \cline { 5 - 6 } & $\begin{array}{c}\text { Cobertura } \\
(\boldsymbol{\%})\end{array}$ & $\begin{array}{c}\text { Altura } \\
(\mathbf{c m})\end{array}$ & $\begin{array}{c}\text { Cobertura } \\
(\boldsymbol{\%})\end{array}$ & $\begin{array}{c}\text { Altura } \\
(\mathbf{c m})\end{array}$ \\
Física & $50,38 \pm 3,39$ & $12,00 \pm 0,46$ & $94,12 \pm 1,56$ & $20,25 \pm 0,9$ \\
Química & $53,50 \pm 3,30$ & $13,50 \pm 0,52$ & $95,87 \pm 1,45$ & $23,12 \pm 0,96$
\end{tabular}

*Medias en la misma columna no difieren, según prueba Duncan $(\mathrm{p}=0,18)$.

aumentó conforme se amplió la distancia de siembra (Cuadro 7).

En la banda, dicha cobertura fue similar entre las plantas sembradas a 0,75 y $1,00 \mathrm{~m}$, mientras que en el centro la cobertura de las parcelas con plantas sembradas a 0,50 y $0,75 \mathrm{~m}$ fue similar. Se puede notar que el porcentaje de malezas en la banda es menor que en el centro (Cuadro 7).

Cuadro 7. Cobertura y altura de malezas ( \pm error estandar) en la banda y en el centro en cuatro distancias de siembra 9 mds. Guápiles, Limón. Costa Rica. 2000-2001.

\begin{tabular}{ccccc}
\hline $\begin{array}{c}\text { Distancia } \\
(\mathbf{m})\end{array}$ & $\begin{array}{c}\text { Cobertura } \\
(\boldsymbol{\%})\end{array}$ & $\begin{array}{c}\text { Error } \\
\text { estandar }\end{array}$ & $\begin{array}{c}\text { Altura } \\
(\mathbf{c m})\end{array}$ & $\begin{array}{c}\text { Error } \\
\text { estandar }\end{array}$ \\
\hline & Banda & & & \\
0,25 & $11,75 \mathrm{c}$ & 1,53 & $19,50 \mathrm{a}$ & 2,46 \\
0,50 & $28,75 \mathrm{~b}$ & 2,90 & $14,50 \mathrm{~b}$ & 1,35 \\
0,75 & $39,50 \mathrm{a}$ & 3,20 & $14,00 \mathrm{~b}$ & 1,52 \\
1,00 & $44,00 \mathrm{a}$ & 3,11 & $13,50 \mathrm{~b}$ & 1,31 \\
& & & & \\
& Centro & & & \\
0,25 & $60,50 \mathrm{c}$ & 4,95 & $20,00 \mathrm{a}$ & 1,92 \\
0,50 & $68,00 \mathrm{~b}$ & 5,35 & $15,50 \mathrm{~b}$ & 1,53 \\
0,75 & $66,50 \mathrm{~b}$ & 5,16 & $16,00 \mathrm{~b}$ & 1,97 \\
1,00 & $75,00 \mathrm{a}$ & 5,74 & $15,00 \mathrm{~b}$ & 1,54 \\
\hline
\end{tabular}

Medias con letra diferente en la misma columna, difieren según prueba Duncan, $(\mathrm{P} \leq 0,05)$.

Las malezas presentaron mayor altura, tanto en la banda $(\mathrm{p} \leq 0,001)$ como en el centro $(\mathrm{p} \leq 0,013)$ en las parcelas cuyas plantas estaban a $0,25 \mathrm{~m}$ entre sí; en la banda, la tendencia fue a una leve disminución de la altura cuando la distancia entre plantas aumentó. Al igual que lo observado con la cobertura, la altura de malezas en la banda fue menor que en el centro (Cuadro 7).

\section{Grado de daño en malezas y cultivo}

En la primera aplicación de glifosato en la banda (15 dda), se observó daño severo (con más del $75 \%$ de tejido necrosado) en las malezas, excepto cinco plantas (una de A. Conyzoides, dos de Colocasia esculenta y dos de Acalypha alopecurioides) en las cuales no se presentó daño. También en la aplicación al centro no se observó daño en cinco plantas de C. esculenta. Después de la cuarta aplicación a la banda (15 dda) se observaron 22 plantas sin síntomas (tres de A. alopecuriodes, 11 de Spermacoce sp y ocho de $C$. ferax) mientras que en el centro se presentaron 20 plantas sin ningún tipo de daño (seis de L. alopecurioides, cinco de C. esculenta y nueve de Stachytarpeta cayenensis).

En la primera aplicación tanto en la banda como en el centro (15 dda), se observaron dos plantas de pejibaye con síntoma grado 1 (leve con dos hojas cloróticas) y una planta con grado 2 (moderado con cuatro hojas cloróticas) y después de la cuarta aplicación (15 dda), se observaron un total de 30 plantas afectadas, de las cuales 28 estaban entre grado 1 y 2 ; otra planta tenía daño 3 (clorosis general) y la última con cuatro (clorosis general con corrugamiento de la candela, tipo roseta). Ninguna de la plantas afectadas murió, sino que se recuperaron dos meses después de la evaluación.

\section{DISCUSIÓN}

\section{Total de especies}

Antes de la siembra el terreno seleccionado tuvo muy poca mecanización, lo cual puede explicar la alta incidencia (95\%) de P. faciculatum (gamalote) junto con un 5\%, de especies de hoja ancha como Sida sp (escobilla) que es una especie leñosa. Al respecto FroudWilliams, citados por Alan et al. (1995) señalan que la labranza reducida tiende a estimular el establecimiento de especies perennes tanto poaceas como leñosas.

Existen factores que activan el banco de semillas de malezas presentes en el suelo; la remoción por medio de la mecanización hace que la semilla de las malezas quede expuesta en niveles superiores y germine (Agüero 1984). También la limpieza o traslado de cobertura vegetal de la superficie mejora la penetración de radiación solar que induce la germinación de semillas latentes (Rojas y de la Cruz 1998).

En este estudio se mecanizó el suelo antes de la siembra para ayudar con un mejor enraizamiento de las plantas de palmito que no tenían adobe. La mecanización 
puede explicar el efecto sobre la activación del banco de semillas en toda el área experimental ya que aumentó el número de especies de malezas uno y seis meses después de la siembra, con respecto a antes de la preparación.

La cobertura de las plantas de palmito a partir del año de edad disminuyó la entrada de radiación solar a estratos inferiores, este factor y el efecto del herbicida probablemente causaron la disminución de especies de malezas 14 mds.

En ese sentido Ramírez (1992) encontró gran efecto de la sombra en el número de especies de malezas en café, mientras que Rojas y de la Cruz (1998) demostraron el efecto de dos herbicidas en la disminución del número de especies de malezas en banano.

\section{Cobertura por grupos y dominancia de especies}

El P. fasciculatum es la principal maleza poacea en plantaciones jóvenes de palma aceitera en Costa Rica (Fernández y Ortiz 1995) y según Corea (1978) es una planta perenne con estolones rastreros y largos, tallos hasta $2 \mathrm{~m}$ de alto con $1 \mathrm{~cm}$ de grosor y hojas que se expanden de 20 a $60 \mathrm{~cm}$ de largo. Las características de esta especie, la principal dentro de las poaceas en este estudio, explican que se extendiera con facilidad (6 mds) formando una gran cobertura junto con otras especies del mismo grupo en las parcelas con control físico, práctica que en presencia de alta luminosidad favorece su dominancia.

En las parcelas con estrategia química el glifosato controló muy bien las especies poaceas y las pocas ciperáceas presentes lo cual influyó en una mayor cobertura de especies con hoja ancha. El glifosato es un herbicida de amplio espectro y controla muy bien especies poaceas (Fernández y Ortiz 1995 ). Con respecto a $P$. fasciculatum, Corea (1978) también obtuvo resultados efectivos en su control con dosis de glifosato entre 0,5 y $1,5 \mathrm{~kg}$ de i.a./ha aplicado en tres épocas $(7,14$ y 21 días después de una chapea).

Dentro del grupo de especies de hoja ancha la especie dominante desde los 6 mds fue Ageratum conyzoides conocida como Santa Lucía. Es probable que sus características y la oportunidad de crecimiento que se le dio (sobre todo en los centros) favorecieron una rápida distribución lo que amplió su cobertura en las parcelas con control químico; en ese sentido, LeRoy et al (1991) la consideran como una de las 200 malezas más importantes a nivel mundial por su amplia distribución y la cantidad de cultivos en que se reporta. Informan, que es una especie invasora debido a que puede producir hasta 40.000 semillas por planta en menos de dos meses.
Es importante mencionar que a partir de los $6 \mathrm{mds}$ en las parcelas con ambas estretegias de control aparecen algunas especies como Drimaria cordata, Lindernia crustacea, Mecardonia procumbens y Stemodia verticilata las cuales tienen una arquitectura y un mecanismo de distribución que les confiere potencial para un manejo diferenciado hasta convertirlas en cobertura de malezas nobles. En ese sentido D. cordata ya ha sido evaluada como cobertura en palmito (Vargas 1995).

En ambas estrategias de control, es posible que la sombra que propiciaron las plantas en las parcelas a alta densidad (0,25 m entre planta) fue la que afectó la cobertura de poaceas y de especies de hoja ancha. Ramírez (1992) en un levantamiento de malezas en café determinó la influencia de la sombra sobre las especies de malezas, y reporta un $93 \%$ de cobertura en café sin sombra, $62 \%$ en condiciones de media sombra y $50 \%$ de cobertura de las especies en plantaciones con sombra, concluyendo además que distanciamientos amplios del café favorecen la proliferación de especies de malezas en diversidad y número.

\section{Cobertura y altura en las bandas y en los centros}

$\mathrm{Al}$ inicio de la investigación (2 mds) los tratamientos estrategias de control y distancias de siembra no causaron efecto alguno sobre la cobertura y la altura de las malezas, eso se explica porque los ciclos de control eran pocos y las plantas de palmito no habían crecido lo suficiente como para afectar con su sombra. Sí es clara la diferencia general que se marca desde un inicio entre la banda y el centro que es independiente de la estrategia o la distancia y obedece al manejo diferenciado que se les dio, o sea se mantuvo siempre menos cobertura y altura de malezas en la banda que en el centro.

Esa idea de manejo diferenciado fue una aproximación práctica porque no existe información de nivel crítico de competencia por malezas en este cultivo y se implementó con la idea de un manejo racional de las mismas; en ese sentido Rojas y de la Cruz (1998) usaron como criterio para realizar los ciclos de aplicación en una evaluación en banano adulto cuando la cobertura superara un $30 \%$ de la parcela.

El manejo físico de las malezas a partir del tercer ciclo se hizo con motoguadaña, lo cual le permitió a $P$. fasciculatum extenderse y formar una cobertura que fue superior en los centros y casi no varió entre densidades.

A partir de los 6 mds y hasta los 14 mds el tamaño y principalmente la cantidad de plantas de palmito, ejercieron un efecto de competencia por espacio y radiación solar que explican la relación observada entre 
la cobertura y la altura de las malezas con las distancias de siembra; al respecto, el manejo de la distancia de siembra es considerado como un método cultural para controlar las malezas (Alan et al. 1995, Gamboa 1999).

\section{Grado de daño en malezas y cultivo}

El control con glifosato, fue bueno sobre la mayoría de malezas. Sin embargo, en algunas especies el control no fue eficiente, tal es el caso de Colocasia esculenta que cuando la superó los $20 \mathrm{~cm}$ de altura y tuvo más de cuatro hojas no mostró síntomas de daño, mientras que, plantas con hasta $10 \mathrm{~cm}$ y dos hojas si fueron controladas. Otras malezas como Ageratum conyzoides y Acalypha alopecurioides no fueron por el herbicida (efecto "sombrilla") cuando la aplicación se hizo a la banda y la altura de otras malezas era de casi $40 \mathrm{~cm}$. En el cuarto ciclo de aplicación las plantas de Stachytarpeta cayenensis, Ludwigia suffruticosa y Spermacoce sp fueron afectadas muy levemente ya que eran muy leñosas y estaban en floración por lo que quizá la dosis de herbicida $(0,5 \%)$ usada en ese ciclo no fue suficiente.

El glifosato es un herbicida sistémico de amplio espectro que afecta al cultivo pero se puede usar si se aplica en forma dirigida a las malezas (Soto y Valverde 1991); lo que significa que en las aplicaciones (principalmente en la banda), no se debe asperjar al cultivo. Sin embargo, si se analizan los datos del cuadro dos, la cobertura y la altura de las malezas en la banda durante la primera y cuarta aplicación pudo incidir en que se levantara la boquilla y se rociara parcialmente algunas plantas. Lo anterior, puede explicar los síntomas de toxicidad por glifosato observados en plantas de palmito que posteriormente se recuperaron. Los síntomas coincidieron con los que se describen para el herbicida (Agüero 1999) y con los observados en una prueba preliminar en la que se rociaron plantas de pejibaye en vivero para determinar los síntomas. Sobre el tema, Agüero et al (1998), hacen una contribución importante demostrando que en banano ciclos consecutivos de aspersión de glifosato, dirigidos a la maleza, con una altura de boquilla adecuada con respecto a los hijos fotosintéticamente activos, no provocaron síntomas de toxicidad.

\section{CONCLUSIONES}

En general, se puede concluir que, en este experimento se observó una gran diferencia entre estrategias de control con respecto al número y cobertura de especies de malezas así como un efecto de la distancia de siembra entre plantas de palmito sobre el crecimiento de las malezas en la banda.

\section{LITERATURA CITADA}

AGÜERO, R. 1984. Evaluación de sistemas de labranza con arroz de secano en rotación con sorgo forrajero. Tesis de posgrado. CATIE. Costa Rica. 49 p.

AGÜERO, R.; PEREZ, L.; GUZMAN, M. 1998. Crecimiento y rendimiento del banano (Musa AAA) bajo ciclos consecutivos de aspersión con glifosato. Agronomía Mesoamericana 9 (2): 105-112.

AGÜERO, R. 1999. Herbicidas que inhiben síntesis de aminoácidos. Apuntes de clase. In Curso: Fisiología y modo de acción de herbicidas. Facultad de Agronomía, Universidad de Costa Rica. Mimeografiado 25p.

ALAN, E.; BARRANTES, U.; SOTO, A.; AGÜERO, R. 1995. Elementos para el manejo de malezas en agroecosistemas tropicales.. Cartago, Costa Rica. Editorial Instituto Tecnológico de Costa Rica. 223p.

BOGANTES, A. 1996. Recomendaciones para la siembra y manejo de palmito de pejibaye (Bactris gasipaes $\mathrm{B}$. K.). Estación Experimental Los Diamantes. (Costa Rica) Hoja Técnica 12 p.

COREA, M. M. 1978. Determinación de dosis y épocas de aplicación de glifosato en el control de Paspalum fasciculatum Willd. Tesis Ing. Agrónomo. Escuela Nacional de Agricultura y Ganadería. Managua, Nicaragua. pp. $5-26$.

FERNANDEZ, O.; ORTIZ, R. A. 1995. Evaluación de herbicidas graminicidas para el combate de gamalote (Paspalum fasciculatum Wild.) en palma aceitera. Agronomía Mesoamericana (6): 15-22.

GAMBOA, C. 1999. Manejo integrado de malezas. In Palmito de pejibaye (Bactris gasipaes K.), su cultivo e industrialización. Editores Jorge Mora y Javier Gaínza. San José, Costa Rica. Editorial U.C.R. pp. 124-132.

LEROY, G.; PLUCKNET, D.;PANCHO, J.; HERBERGER, J. 1991. Ageratum conyzoides L. and Ageratum houstonianum Mill. The world's worst weeds. Distribution and Biology. Krieger Publishing Co. Malabar, Florida. pp. 46-151.

MORA, J. 1989. Densidades de siembra para producción de palmito. Universidad de Costa Rica. Serie técnica Pejibaye 1 (1): 10-12.

MORA, J.; BOGANTES, A.; ARROYO, C. 1999. Culivares de pejibaye para palmito. Palmito de pejibaye (Bactris gasipaes K.). su cultivo e industrialización. Editores Jorge Mora y Javier Gainza. Editorial U.C.R. San José, Costa Rica. pp. 41-47.

PITTY, A.; MUÑOZ, E. 1991. Guía práctica para el manejo de malezas. Escuela Zamorano. Honduras. 222 p. 
RAMIREZ, A. R. 1992. Distribución de malezas en cafetales del Salvador. In V Simposio Latinoamericano sobre caficultura; 20-22 octubre. IICA. San Salvador. El Salvador. $30 \mathrm{p}$.

ROJAS, B. M.; de la CRUZ, R. 1998. Control de malezas en banano (Musa spp.) mediante hojarazca del cultivo. Manejo Integrado de Plagas (49): 58-67.

SOTO, A; AGÜERO, R. 1992. Combate químico de malezas en arroz. San José, Costa Rica. Editorial Universidad de Costa Rica. 81 p.

SOTO, A.; VALVERDE, B. 1991. Los herbicidas. Propiedades físico-químicas, clasificación y mecanismos de ac- ción. San José. Costa Rica. Editorial Universidad de Costa Rica. pp 62-63.

VALVERDE, L. ; UGALDE, M. 1990. Estudio detallado de los suelos de la Finca Experimental Los Diamantes. Informe de presentaciones taller información de suelos. Ed. Wielemaker W. Convenio CATIE-MAG-UAW. Guápiles, Costa Rica pp 15-17.

VARGAS, A. 1995. Evaluación preliminar de coberturas vegetales vivas en el cultivo de pejibaye para palmito (Bactris gasipaes K.) en relación a su efecto sobre la fertilidad del suelo, productividad del cultivo e incidencia de malezas. CORBANA 20 (44): 25-32. 